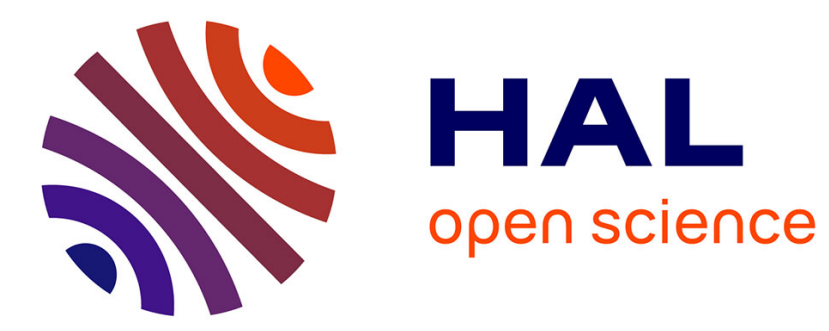

\title{
Stratigraphy of a diamond epitaxial three-dimensional overgrowth using doping superlattices
}

\author{
F Lloret, A Fiori, D Araujo, D. Eon, M.P. Villar, E. Bustarret
}

\section{To cite this version:}

F Lloret, A Fiori, D Araujo, D. Eon, M.P. Villar, et al.. Stratigraphy of a diamond epitaxial three-dimensional overgrowth using doping superlattices. Applied Physics Letters, 2016, 108 (18), pp.181901. 10.1063/1.4948373 . hal-01319640

\section{HAL Id: hal-01319640 \\ https://hal.science/hal-01319640}

Submitted on 24 May 2016

HAL is a multi-disciplinary open access archive for the deposit and dissemination of scientific research documents, whether they are published or not. The documents may come from teaching and research institutions in France or abroad, or from public or private research centers.
L'archive ouverte pluridisciplinaire HAL, est destinée au dépôt et à la diffusion de documents scientifiques de niveau recherche, publiés ou non, émanant des établissements d'enseignement et de recherche français ou étrangers, des laboratoires publics ou privés. 


\title{
Stratigraphy of a diamond epitaxial 3D overgrowth using doping superlattices
}

\author{
F. Lloret ${ }^{1}$, A. Fiori², D. Araujo ${ }^{1}$, D. Eon ${ }^{3,4}$, M.P. Villar ${ }^{1}$, and E. Bustarret ${ }^{3,4}$ \\ ${ }^{1}$ Departamento de Ciencias de los Materiales e IM y QI, Universidad de Cádiz, 11510 Cádiz, Spain \\ ${ }^{2}$ National Institute for Materials Science, 1-1 Namiki, Tsukuba, Ibaraki 305-0044, Japan \\ ${ }^{3}$ Université Grenoble Alpes, Institut NEEL, F-38042 Grenoble, France \\ ${ }^{4}$ CNRS, Institut NEEL, F-38042 Grenoble, France
}

The selective doped overgrowth of 3D mesa patterns and trenches has become an essential fabrication step of advanced monolithic diamond-based power devices. A novel methodology is proposed here, combining the overgrowth of plasma-etched cylindrical mesa structures with the sequential growth of doping superlattices. The latter involve thin heavily boron doped epilayers separating thicker undoped epilayers in a periodic fashion. Beside the classical shape analysis under the scanning electron microscope relying on the appearance of facets corresponding to the main crystallographic directions and their evolution toward slow growing facets, the doping superlattices were used as markers in oriented cross-sectional lamellas prepared by Focused Ion Beam and observed by Transmission Electron Microscopy. This stratigraphic approach is shown here to be applicable to overgrown structures where faceting was not detectable. Intermediate growth directions were detected at different times of the growth process and the periodicity of the superlattice allowed to calculate the growth rates and parameters, providing an original insight into the planarization mechanism. Different configurations of the growth front were obtained for different sample orientations, illustrating the anisotropy of the 3D growth. Dislocations were also observed along the lateral

growth fronts with two types of Burger vector: $b_{01 \overline{1}}=\frac{1}{2}[01 \overline{1}]$ and $b_{112}=\frac{1}{6}[112]$. Moreover, the clustering of these extended defects in specific regions of the overgrowth prompted a proposal of two different dislocation generation mechanisms. 
In the quest for power electronic devices sustaining ever higher reverse blocking voltages and forward currents, buried heavily boron doped $\left(\mathrm{p}^{+}\right)$diamond layers have been shown recently ${ }^{1}$ to reduce markedly the on-state resistance $\left(R_{o n}\right)$ of pseudo-vertical single crystal diamond Schottky diodes, to the point where an impressive Baliga's power figure of merit of $244 \mathrm{MW} / \mathrm{cm}^{2}$ could be reached. This compared favorably to the results obtained with smaller bandgap semiconductors, or to the results using a vertical diamond geometry. Similarly, heavily phosphorus doped $\left(\mathrm{n}^{+}\right)$epitaxial layers have been introduced in the lateral $\mathrm{p}-\mathrm{n}$ junctions ${ }^{2}$ of diamond field-effect transistors ${ }^{3}$. These results illustrated the advantages of controlling heavily doped/undoped interfaces, and confirmed that the more complicated architectures which have been envisioned for some time ${ }^{4}$ were becoming feasible. Such advanced designs, where the doping level or doping type are modulated along both the lateral and the vertical directions, rely on an improved control of the selective 3D overgrowth of dry-etched mesa and trenches ${ }^{5-8}$, an issue solved a few decades ago in the case of silicon ${ }^{9}$.

Diamond 3D growth mechanisms by chemical vapor deposition (CVD) or at high-pressure and high-temperature (HPHT) have been largely studied ${ }^{10-15}$ and the models developed are well known and accepted. These models are mainly based on the relative growth velocities of four low index crystal planes: $\{100\},\{110\},\{111\}$ and $\{113\}$. The value of the velocity ratios (the so-called growth parameters) describing the global morphology of the crystal during the growth process allows to predict their final shape. Because of the good agreement observed between such 3D models and the experimental results, crystal shapes have been used to determine the growth parameters. As shown below in the case of sample \#1, this method may be tentatively extrapolated to the overgrowth of mesa patterns, and the shapes of faceted structures interpreted in order to estimate the growth parameters.

However, the overgrowth of 3D structures such as mesa and trenches etched from the flat surface of a substrate implies volumetric constraints and microscopic roughness generating additional strain at edges and corners. The resulting tensile and compressive forces lead to defect formation and non-expected parasitic growth directions, preventing a clear faceting and a simple use of the above-described models. Moreover, lateral growth fronts originating in neighboring mesa patterns will coalesce at some point, leading to a planarization of the structure that may be the main objective of the overgrowth, but precludes a straightforward a posteriori domain shape analysis.

In order to study the intermediate stages of non-faceted or planarized overgrown diamond structures, a new approach is here proposed, which involves heavily doped nm-thick sublayers used as time markers in a stratigraphic 
configuration, similar to previous studies of stacks of SiGe alloys ${ }^{16}$. As shown below for sample \#2, the borondoped sublayers are easily distinguishable by transmission electron microscopy (TEM) in the conventional diffraction contrast mode ${ }^{17}$, and act as milestones of the growth plane orientation as growth proceeds. This method was designed to address both the issue of defect generation brought about by strong doping modulation, and that of progressive planarization of mesa or trench structures upon epitaxial overgrowth.

A deep-UV light bilayer mask resist was employed to draw open disks of 1-50 microns in diameter on two Ibtype (100)-oriented $3 \times 3 \mathrm{~mm}^{2}$ diamond substrates by UV laser lithography. These open disks were filled by nickel deposited by electron beam evaporation in a high vacuum chamber to obtain round solid masks. After the lift-off of the bilayer resist, the surface of diamond substrates decorated by nickel disks was etched away. The etching took place in a deep reactive-ion etching machine and the oxygen plasma conditions were optimized to obtain a high aspect ratio. Approximately 700 nanometers of diamond were etched way, forming a pattern of diamond cylinders topped with nickel. Afterward, nickel was removed in a boiling aqua regia solution, and etched diamond substrates were washed in a boiling mixture of nitric, sulfuric, and perchloric acids.

The etched diamond substrates were then overgrown by microwave plasma-assisted chemical vapor deposition (MPCVD) in a NIRIM-type quartz wall reactor. In the case of sample \#1, a single undoped layer was overgrown. Methane and oxygen were diluted in purified hydrogen carrier gas, in relative ratios $\mathrm{CH}_{4} / \mathrm{H}_{2}=1 \%$ and $\mathrm{O}_{2} / \mathrm{H}_{2}=0.25 \%$, well known to yield undoped CVD diamond ${ }^{18}$. In the case of sample \#2, a stack of nine periodic alternates of undoped and boron-doped $\left(10^{20} \mathrm{at} / \mathrm{cm}^{3}\right)$ diamond layers was overgrown. The gas composition in the deposition chamber was switched automatically following a preset periodic flow sequence. The gas mixture yielding heavily boron-doped layers was composed of methane and diborane diluted into purified hydrogen, with the following molar ratios: $\mathrm{CH}_{4} / \mathrm{H}_{2}=0.5 \%$ and $\mathrm{B}_{2} \mathrm{H}_{6} / \mathrm{CH}_{4}=14000 \mathrm{ppm}^{18}$. The growth of the undoped layer was undertaken under conditions similar to those of sample \#1, after a 30 seconds hydrogen flush at high flow rate ( $2 \mathrm{slm})$. The microwave power and chamber pressure were kept constant at $400 \mathrm{Watt}$ and 33 Torr throughout the whole process, without interrupting the plasma.

The topography of overgrown cylinders was characterized by an orthogonal secondary electron mapping (inlens SE-SEM), by a stylus 1D profiler, and by an optical interferential 3D profiler. Focused ion beam (FIB) prepared lamellas of sample \#2 have been prepared for cross sectional transmission electron microscopy (TEM) observations 
using a dual beam FEI QUANTA 200 3D microscope and following the lift-out method ${ }^{19}$. TEM preparations were carried out on disks with the same initial diameter $(\sim 50 \mu \mathrm{m})$. Various lamellas with a thickness of around $80 \mathrm{~nm}$ have been extracted within radial planes parallel to the [010] and [011] directions. SEM observations were carried out at $5 \mathrm{kV}$ into a FESEM Zeiss Ultra plus microscope while TEM diffraction contrast study was realized with a $120 \mathrm{kV}$ JEOL 1200 EX TEM microscope along the $<011>$ and $<001>$ zone axis. $\times 7500$ and $\times 12000$ magnification in bright field (BF) and dark field (DF) modes were used in order to have an overall view of the doped layers in the samples. Weak beam (WB) mode under two beam conditions was used for the study of defects.

The overgrowth of sample \#1 yielded a single undoped layer. Fig. 1(a) shows a SEM micrograph of mesa-etched cylinders whose diameter was 4 microns, and Fig. 1(b) showed the same place once overgrown. The new contours of mesa-etched cylinders upon overgrowth have lost the circular geometry; the final shape appeared polyhedral (see Fig. 1(b)). A first observation suggested the existence of a (100) top facet accompanied by others such as $\{110\}$ and $\{311\}$ localized at the edges and corners of the overgrown cylinders. 

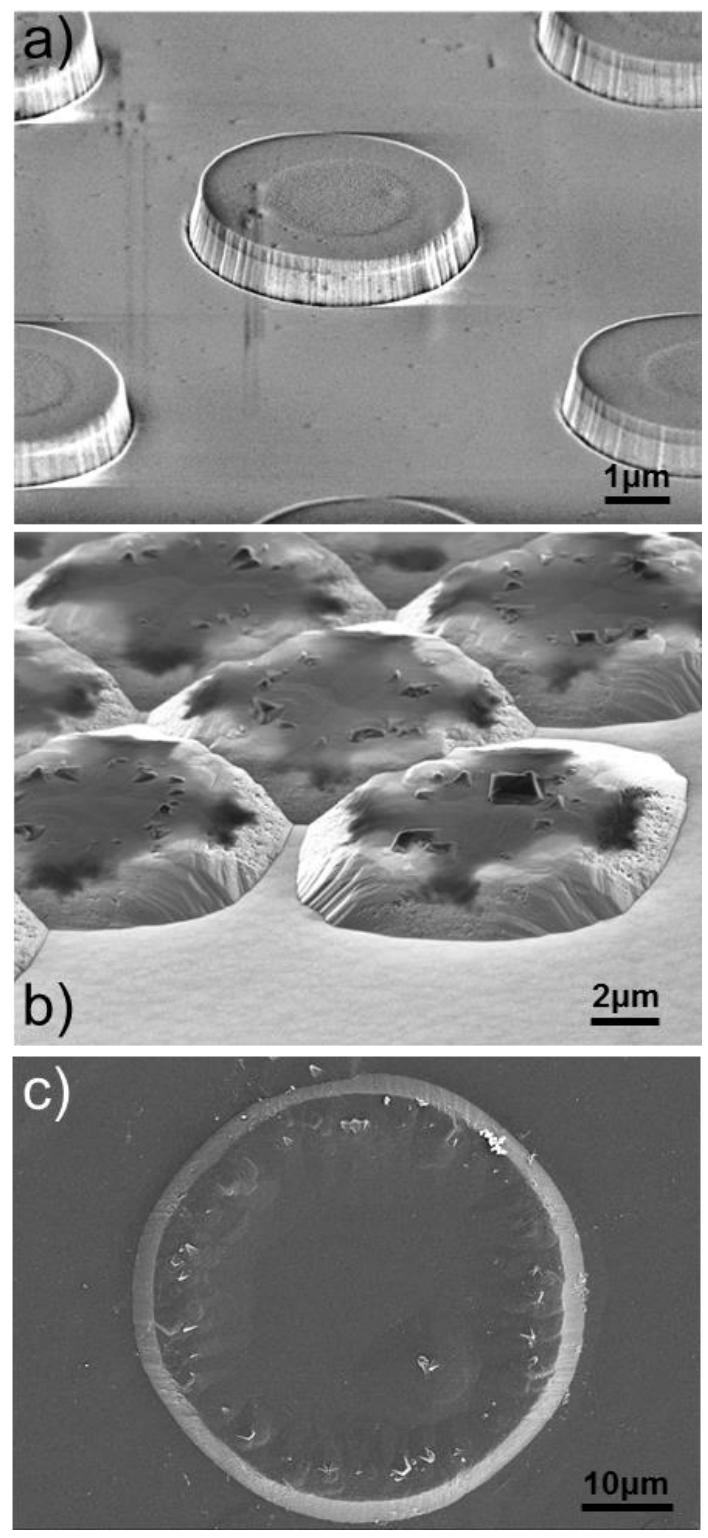

FIG. 1. SEM images of sample \#1 taken before (a), after (b) a 70 min overgrowth by an undoped diamond layer and the superlattice overgrowth on the sample \#2 (c).

The crystallographic orientation of facets has been determined geometrically by measuring lengths, distances between edges, and tangent angles by assuming a (100) plane at the top of overgrown cylinders. Fig. 2(a) displays the crystallographic orientation of facets determined geometrically for sample \#1. Facets along $\{311\}$ and $\{110\}$ planes were identified on borders. Cut edges with second order facets were found on $\{110\}$ sides. These secondary facets could be tentatively ascribed to crystallographic planes $\{210\}$ and $\{310\}$. The small size of these secondary facets does not allow to claim the exact plane orientation, and to point out the limits of the geometrical determination for the present dimensions and spacings. 
The height of overgrown cylinders was the same as before deposition; this confirmed the top of the overgrown cylinders followed the initial (100) plane. The vertical growth rate (100) has been measured on the cross section of a 10-micron-thick layer grown under the same conditions ${ }^{18}$. Growth rates of facets have been estimated following a simple trigonometry, illustrated in Fig. 2(b), and summarized in table I. In this method, we considered that the adjacent side was the facet altitude, and the opposite side was the growth rate. The distance separating the mesaetched cylinder and the intersection of the facet with the original (100) plane gave an orthogonal projected length of the facet.

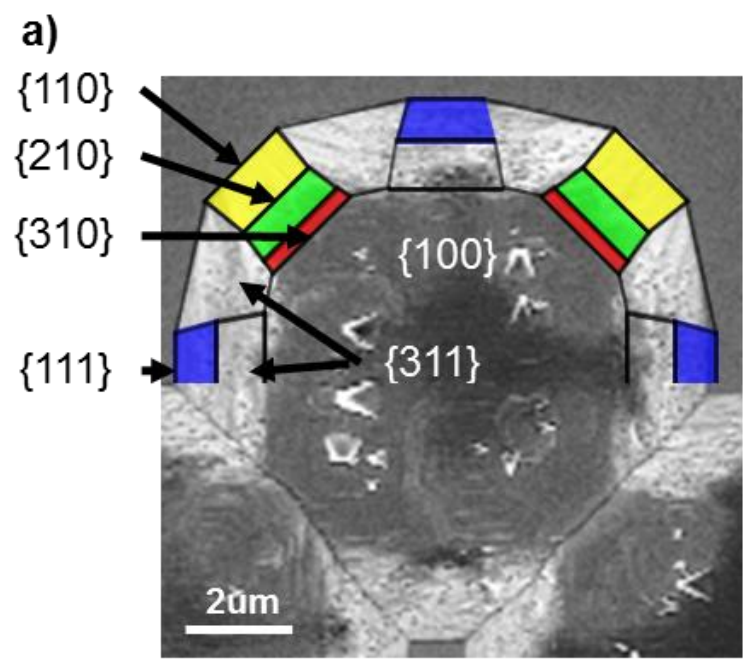

b)



FIG. 2. (a) Top view of an overgrown cylinder with indexed facets superimposed. Each color corresponds to a family of planes. (b) Schematic cross-section of an overgrown cylinder used to calculate the growth rate of the (111) plane. Solid and dash-dotted lines represent the final surface and the initial surface at the edge of the overgrown cylinders respectively.

Under the growth conditions of sample \#1, the planes family slowest to grow after the (100) was the $\{311\}$. These types of planes in diamond were called stepped faces due to the large number of terrace edges, and were the 
most stable. However, the stability of such faces cannot be generalized in our case, because of the number of defects present at the wells of the mesa-etched cylinders, and because of a typical trenching effect. Twinning may also arise on an edge of a face, or on the faces of a re-entrant etch pits, and emerge at the surface. This has been observed on sample \#1, where twinning hillocks are describing a circle following the top border of mesa-etched cylinders.

The growth rate of $\{111\},\{110\}$, and $\{311\}$ faces normalized to the displacement of $\{100\}$ surfaces gave three growth parameters as follows ${ }^{11}$ :

$$
\begin{aligned}
& \alpha=\sqrt{3} \frac{V_{\{100\}}}{V_{\{111\}}} \\
& \beta=\sqrt{2} \frac{V_{\{100\}}}{V_{\{110\}}} \\
& \gamma=\sqrt{11} \frac{V_{\{100\}}}{V_{\{113\}}}
\end{aligned}
$$

Taking (100) as the orientation of the top surface, the growth anisotropy factor was defined as

$$
\mathcal{A}=\frac{V_{[010]}}{V_{[100]}}=\frac{V_{[001]}}{V_{[100]}}
$$

The shape of twinning found at the top of overgrown cylinders was helpful to estimate the $\alpha$ parameter, as reported by C. Wild et al. ${ }^{10}$. From these twinning, we geometrically found $\alpha \approx 1.55$. Then, we were able to estimate $\beta \approx 1.55$, and $\gamma \approx 0.9$. According to Silva et al. ${ }^{11}$, these values correspond to the criteria " $\alpha=\beta, \alpha>1, \gamma<11 \alpha / 5$ " which predicted the disappearance of $\{111\}$ faces on the top side with the dominance of $\{311\}$ over $\{111\}$ faces. With an anisotropy factor close to 6.65 , the contours of overgrown cylinders after an extended overgrowth would bordered with $\{113\}$ facets. This would involve the disappearance of $\{110\}$ faces and secondary faces too.

The present method is a very simple way of estimating growth rates based on the shape of well-faceted patterns. It does not require any cross section analysis nor destructive characterization while providing valuable information about the growth. However, it has at least three limitations that should be kept in mind: (i) as previously mentioned for the case of secondary faces, facets are not always easy to identify, especially for large disks and short growth durations. (ii) The method assumes that the growth follows just one low index growth direction. It does not consider possible intermediate planes. And (iii) it is not obvious to deduce where the intersection of the facet with the original plane should be fixed, a source of error on the numerical values. 
The first limitation was illustrated by the case of sample \#2, where SEM observations showed cylinders with non-faceted lateral faces (Fig. 1(c)). In this case, the diamond growing at the top of mesa-etched cylinders kept the same growth direction, while the diamond growing on the sidewalls had a growth direction that changed with time. The variation of the growth direction could be related to strain gradients, crystal defects generation and coalescence, but the shape analysis does not provide any quantitative clue about the growth mechanisms. Fortunately, thanks to the presence of doped layers, an alternative method based in cross-section observations allowed to reveal such internal growth features of the overgrown cylinders. To this end, two cross-section lamellas were extracted from the sample \#2. The identification of growth features was guided by the contrast associated to the doping superlattice, which made the stratigraphy possible. Therefore, a first cross-section lamella has been cut along [011] , and a second along [010]. These lamellas have been analyzed by TEM in the bright field (BF) (Fig. 3(a)) and dark field (DF) modes (Fig. 3(b)), using different reflections of the same pole to reveal boron-doped layers and dislocations.
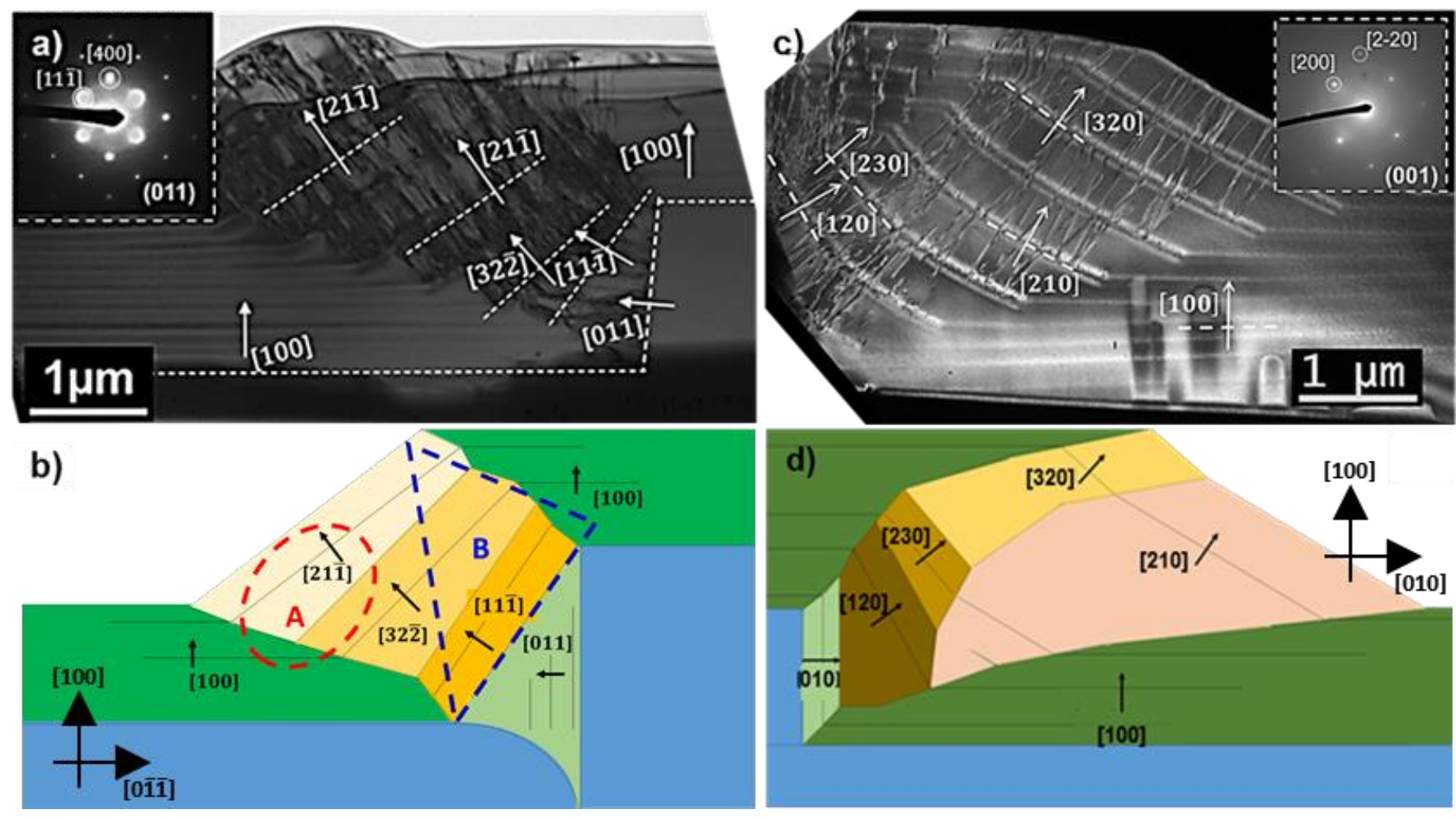

FIG. 3. (a) Bright field micrograph of a cross-section made along the [011̄] direction, observed at (011) pole. (c) Dark field micrograph of a cross-section made along the [010] direction observed at (001) pole. In these micrographs, white dashed lines and arrows indicate the position of $\mathrm{p}^{+}$layers and growth directions respectively. (b) and (d) show schematic views of the groups of growth planes identified by color deduced from TEM observations of [011] and [010] direction respectively. In (b), the regions where A and B types of dislocations predominate are delimited by broken lines. 
Fig. 3(a) shows a bright-field (BF) TEM micrograph of the cross-section lamella made along the $\{011\}$ plane. That micrograph was recorded with the lamella oriented in the (011) zone axis, as shown in the diffraction pattern (see the corresponding inset). To guide the eyes, white dashed lines defined the position of the surface prior to overgrowth. It highlighted the sidewall and top of a mesa-etched cylinder. On the BF TEM image, boron-doped layers of the doped superlattice appeared in dark contrast. Fig. 3(c) shows a dark-field (DF) TEM image of the cross-section lamella prepared along the $\{010\}$ plane and recorded in the (001) zone axis (see the corresponding diffraction pattern in inset). On the DF TEM micrograph, boron-doped layers appeared as pairs of white bright lines. The reason to observe two white lines for one boron-doped layer has been associated to interface strain in the literature $^{20}$. The effect of strain on the contrast has been often observed on TEM micrographs of quantum dots especially. The median position between such two white lines has been taken as a reference to measure the gap between boron-doped layers. In the [100] direction, boron-doped layers were parallel with a constant spacing, which confirmed the periodic alternate deposition of the doped superlattice.

TABLE I. Growth rates (in $\mathrm{nm} / \mathrm{min}$ ) of facets estimated in sample \#1 (single undoped layer), and calculated \#2 (doped superlattice).

\begin{tabular}{|c|c|c|c|c|c|c|c|c|c|c|c|c|}
\hline & (120) & (110) & (210) & (230) & (310) & $(320)$ & $(\mathbf{1 0 0})$ & $(31 \overline{1})$ & $(21 \overline{1})$ & $(32 \overline{2})$ & $(\mathbf{1 1 \overline { 1 }})$ & $(\mathbf{0 1 \overline { 1 }})$ \\
\hline$\# 1$ & - & 38.9 & 30.0 & - & 26.2 & - & 7.3 & 21.4 & - & - & 32.6 & - \\
\hline \#2 & 36.9 & - & 34.6 & 43.0 & - & 40.6 & 14.3 & - & 25.4 & 23.2 & 8.0 & 27.1 \\
\hline
\end{tabular}

In both cross-sections, high densities of defects were present in the diamond located at the sidewall of the plasmaetched cylindrical mesa. At the opposite, almost none of such defects were located at top surface, following the [100] direction. Two main factors could explain these defects: (i) the influence of the growth orientation in the defects generation ${ }^{21,22}$, and (ii) the tension accumulated in boron-doped layers steps corners ${ }^{23}$. However, threading dislocations were useful to follow the growth direction of diamond at the sidewalls of the mesa-etched cylinder, because threading dislocations propagate along the growth direction ${ }^{24}$. White dashed lines have been drawn on the TEM images of Fig. 3, with white arrows labelling these growth orientations. This pointed out an evolution of the growth direction, and a tendency to planarization. Schematic drawings of cross-sections made along [011̄] and [010] directions (Fig. 3(b) and Fig. 3(d) resp.) highlighted the different orientations of growth planes. In these maps, colored regions correspond to successive growth directions. The boundaries between these regions are marked by dashed lines, and straight lines indicated the orientations of boron-doped layers. These maps exposed the progress of the growth from [011] to [211] direction, which was tilted at approximately $34^{\circ}$ with respect to the (100) plane. 
In addition, the position of boron-doped layers acted as a time reference, allowing the calculation of growth rates for each direction. The growth rates summarized in Table I showed that the growth was faster for the terminal orientations: $\{210\}(\sim 35 \mathrm{~nm} / \mathrm{min})$, and $\{320\}(\sim 41 \mathrm{~nm} / \mathrm{min})$. The evolution of growth orientation observed in sample \#1 was partially reproduced in sample \#2. For example, the $\{311\}$ facet in sample \#1 seemed to be replaced by $\{322\}$ and $\{211\}$ second order facets in sample \#2, but with unchanged growth rate. Similarly, the $\{110\}$ facet in sample \#1 was substituted by $\{120\}$ and $\{210\}$ in sample \#2. One-step beyond, the $\{310\}$ second order facet in sample \#1 could be viewed as $\{320\}$ and $\{230\}$ third order facets found in sample \#2. These results confirm the validity of the first method for well faceted samples.

Moreover, the influence of boron-doped layers on the generation of dislocations is certain (boron inclusion, strain). Different types of defects have been identified in the sample \#2 by applying the invisibility criterion on TEM micrographs ${ }^{25}$. Two kinds of Burger vectors have been found: $\mathbf{b}_{\mathbf{1 1 2}}=\frac{1}{6}[112]$, and $\mathbf{b}_{\mathbf{0 1}} \overline{\mathbf{1}}=\frac{1}{2}[01 \overline{1}]$ corresponding to type A and B respectively. These dislocations aggregated in different regions, delimited by a red circle and a blue triangle in Fig. 3(b) for type A and B defects respectively. Depending on the Burger vectors, defects seemed to have different mechanism of generation. We noticed the type A extended defects were occurring closer to the final surface, while type B dislocations were generated in the region closer to the sidewall of the mesa-etched cylinder. Tentatively, the generation of type B dislocations would be enhanced by the tensile stress present at the corners of the mesa-etched cylinder. Type A defects could be generated by the inclusion of the boron atoms ${ }^{21}$.

The overgrowth of mesa-etched diamond cylinders combined with a stratigraphic approach is a powerful tool to evaluate the growth rates along different crystallographic directions. In the first example provided here, faceted overgrowth shows that the top face (100) was the slowest to grow. Faces $\{111\}$ were visible only at twinning emergence, and $\{113\}$ faces were presumed to border the sample at the equilibrium. $\{110\}$ faces appeared in ephemeral transition phases at the edges of mesa-etched structures. The difference in the growth rate between those faces confirmed the tendency to obtain a flat top surface and the reduction of defects height.

Overgrowth realized with doping superlattices adds supplementary insight into the growth and defect generation mechanisms. The growth time sequences is in this way easily visualized by the stratigraphic structure of TEM crosssections observation. A progressive change in the growth directions was observed at intermediate steps showing a 
tendency of planarization. In addition, the use of embedded doped layers made it possible to measure the ratio of growth rates with a high accuracy and to obtain two growth parameters: $\alpha=3.1$ and $\beta=0.75$.

This method allows to follow step by step at a nm-scale the 2D and 3D growth dynamics. However, the presence of boron atoms may generate extra defects, which should affects standard growth mechanisms. These defects, in a high density concentration, may have influence on the growth plane orientation. In order to minimize the effect of the boron atom inclusion, the boron concentration should be minimized. However, the boron doping level must be high enough to introduce a compositional contrast on TEM micrographs. In this work, plenty of threading dislocations have been observed. The study of these defects suggested a stress accumulated at the corner of the mesa structures, and generation centers located inside highly doped layers.

This work was made possible through grants from the Spanish Ministry of Economy and Competitiveness (TEC201454357-C2-2-R, HiVolt-nano project) and from the European H2020 Program (SEP-210184415, GreenDiamond project).

A. Traoré, P. Muret, A. Fiori, D. Eon, E. Gheeraert, and J. Pernot, Appl. Phys. Lett. 104, 052105 (2014).

H. Kato, T. Makino, M. Ogura, N. Tokuda, H. Okushi, and S. Yamasaki, Appl. Phys. Exp. 2, 055502 (2009).

3 T. Iwasaki, J. Yaita, H. Kato, T. Makino, M. Ogura, D. Takeuchi, H. Okushi, S. Yamasaki, and M. Hatano, IEEE El. Dev. Lett. 35, 241 (2014).

$4 \quad$ R.S. Balmer, I. Friel, S.M. Woolard, C.J.H. Wort, G.A. Scarsbrook, S.E. Coe, H. El-Hajj, A. Kaiser, A. Denisenko, E. Kohn, and J. Isberg, Phil. Trans. R. Soc. A 366, 251 (2008).

5 Y. Ando, T. Kamano. K. Suzuki, and A. Sawabe, Jap. J. Appl. Phys. 51, 090101 (2012).

6 Y. Hoshino, H. Kato, T. Makino, M. Ogura, T. Iwasaki, M. Hatano, and S. Yamasaki, Phys. Status Solidi A 209, 1761 (2012).

7 D. Takeuchi, T. Makino, H. Kato, M. Ogura, N. Tokuda, T. Matsumoto, D. Kuwabara, H. Okushi, and S. Yamasaki, Phys. Status Solidi A 10, 2251 (2014).

8 K. Sato, T. Iwasaki, Y. Hoshino, H. Kato, T. Makino, M. Ogura, S. Yamasaki, S. Nakamura, K. Ichikawa, A. Sawabe, and M. Hatano, Jap. J. Appl. Phys. 53, 05FP01 (2014).

$9 \quad$ M.R. Goulding, Mat. Sci. Eng. B-Solid. 17, 47 (1993).

10 C. Wild, R. Kohl, N. Herres, W. Mullersebert, and P. Koidl, Diam. Relat. Mater. 3, 373 (1994).

11 F. Silva, X. Bonnin, J. Achard, O. Brinza, A. Michau, and A. Gicquel, J. Cryst. Growth 310, 187 (2008).

12 F. Silva, J. Achard, X. Bonnin, A. Tallaire, O. Brinza, and A. Gicquel, Phys. Stat. Sol. A 203, 3049 (2006).

13 C.C. Battaile, D.J. Srolovitz, and J.E. Butler, J. Cryst. Growth 194, 353 (1998).

14 A. Tallaire, J. Achard, O. Brinza, V. Mille, M. Naamoun, F. Silva, and A. Gicquel, Diam. Relat. Mater. 33, 71 (2013).

15 A. Bogatskiy, and J.E. Butler, Diam. Relat. Materials 53, 58 (2015).

16 L. Vescan, Mater. Sci. Eng. B 28, 1 (1994).

17 D. Araújo, M.P. Alegre, A.J. García, M.P. Villar, E. Bustarret, P. Achatz, P.N. Volpe, and F. Omnès, Phys. Stat. Solidi C 8, 1366 (2011).

18 G. Chicot, T.N. Tran Thi, A. Fiori, F. Jomard, E. Gheeraert, E. Bustarret, and J. Pernot, Appl. Phys. Lett. 101, 162101 (2012).

19 M. Sugiyama, and G. Sigesato, J. Electron. Microsc. 53, 527 (2004).

20 M. Gutierrez, M. Herrera, D. González, R. García, and M. Hopkinson, Appl. Phys. Lett. 88, 193118 (2006).

21 M.P. Alegre, D. Araújo, A. Fiori, J.C. Pinero, F. Lloret, M.P. Villar, P. Achatz, G. Chicot, E. Bustarret, and F. Jomard, Appl. Phys. Lett. 105, 173103 (2014). 
F. Lloret, D. Araujo, M.P. Alegre, J.M. Gonzalez-Leal, M.P. Villar, D. Eon, and E. Bustarret, Phys. Stat. Sol. A 212, 2468 (2015).

23 R.M. Erasmus, R.D. Daniel, and J.D. Comins, J. Appl. Phys. 109, 013527 (2011).

24 X.F. Zhang, and Z. Zhang, Progress in Transmission Electron Microscopy: Concepts and Techniques, edited by G. Ertl, R. Gomer, H. Lüth, and D. L. Mills, (Springer, Berlin, 2001) p.263.

25 H.J. Scheel, P. Capper, and P. Rudolph, Crystal Growth Technology: Semiconductors and dielectrics, edited by. H.J. Scheel, and P. Capper, (Wiley-VCH, Weinheim, 2010) p.141. 